\title{
Recent Development in Three and Four Dimension Fetal Echocardiography
}

\author{
Rabih Chaoui $^{\mathrm{a}}$ Alfred Abuhamad $^{\mathrm{b}}$ Juliana Martins $^{\mathrm{b}} \quad$ Kai Sven Heling $^{\mathrm{a}}$ \\ ${ }^{a}$ Center for Prenatal Diagnosis and Human Genetics, Berlin, Germany; ${ }^{\mathrm{b}}$ Division of Maternal-Fetal Medicine of the \\ Department of Obstetrics and Gynecology at Eastern Virginia Medical School, Norfolk, VA, USA
}

\section{Keywords}

Fetal echocardiography · Spatial temporal image correlation - Color doppler · Matrix transducer . Three-dimensional ultrasound · Live 4D · Biplane · Automated ultrasound · Artificial intelligence

\begin{abstract}
Since its introduction $>15$ years ago, the use of spatial and temporal image correlation (STIC) technology has contributed substantially to fetal echocardiography. Moreover, significant advances have occurred in 3- and 4-dimensional (3D/4D) echocardiography over the past several years including the matrix probe along with advances in gray scale and color Doppler post processing, resulting in improved display of ultrasound images. In this article, we provide examples to show these recent developments including the use of color Doppler with STIC in the glass-body mode and the matrix probe thus enabling the demonstration of cardiac anomalies of the 4-chamber-view and great arteries. The use of the matrix probe allows the examination of cardiac structures in 2 orthogonal planes simultaneously, which can help in display of anatomy side by side (Biplane mode). In addition, the rapid image reconstruction of the matrix probe allows for the display of live 4D and the rapid acquisition of a STIC volume. The display of multiplanar images of the heart in 3D/4D has also been used to automate the display of ultrasound images, resulting in standardiza-
\end{abstract}

tion of the image display and thus minimizing the operation dependency of the ultrasound examination. Future addition of image recognition software can also provide assistance in image review.

(c) 2019 S. Karger AG, Basel

\section{Introduction}

Fetal echocardiography is an ultrasound examination of the fetal heart, which includes fetal anatomic ultrasound planes of the upper abdomen, the 4 chamber view, the 5 chamber view, the short axis view, the 3-vessel-trachea, and if needed, longitudinal planes of the aortic arch, the ductal arch, and the systemic veins $[1,2]$. Improved diagnostic accuracy of fetal echocardiography is attained by the application of gray scale and color Doppler ultrasound in order to demonstrate blood flow in the cardiac chambers and great vessels. While the atria, ventricles, and atrioventricular valves are simultaneously visualized in the 4-chamber-view, the great vessels are assessed in the left and right outflow tracts thus demonstrating their origin, course, and spatial relationships. Documentation of the fetal echocardiography examination is required and includes storing of still images and movie clips of cardiac anatomy. Review of the fetal echocardiography study at a later time is thus limited to the movie clips and still image obtained by the examiner.

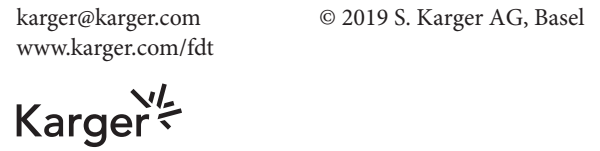


Fig. 1. STIC volume acquisition in color Doppler (a) and in monochromic color Doppler (b) in a longitudinal view of a normal fetus showing the heart and abdominal vessels. Note the the ductus venosus and the inferior vena cava as they connect with the subdiaphragmatic vestibulum. The 2 left pulmonary veins are shown posterior to the left atrium. a One light source (lower right corner) was used and in (b) 3 light sources. AO, aorta; DV, ductus venosus; IVC, inferior vena cava; PV, pulmonary veins.
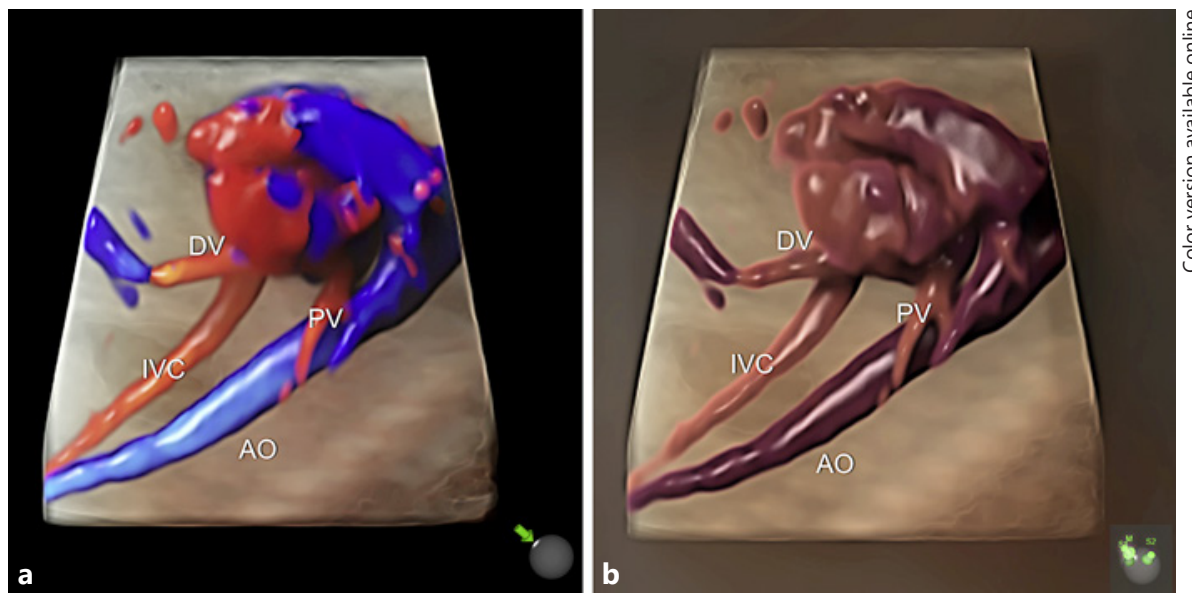

The introduction of 3- and 4-dimensional (3D/4D) ultrasound, especially the spatial and temporal image correlation (STIC) about 15 years ago [3], added several advantages to fetal echocardiography including the ability to extract high number of ultrasound planes from $3 \mathrm{D} / 4 \mathrm{D}$ volumes. Incorporating 3D/4D in fetal echocardiography has been used to display surface mode images with 2-dimensional (2D) and color Doppler [4, 5], to extract single or multiple planes in tomographic view $[6,7]$, thus enabling the offline examination of the heart, a technique used in telemedicine $[8,9]$. Algorithms have been developed on how to extract planes out of a 3D/4D volume; an approach helpful in teaching fetal echocardiography, especially the outflow tracts $[4,5]$. Furthermore, 3D/4D allows for volume calculations of cardiac chambers, which is used to accurately assess cardiac volumes in systole and diastole [10]. In the past several years, novel applications of 3D/4D in fetal echocardiography emerged primarily in 3 different aspects: Color Doppler rendering, the matrix probe with biplane, or X-plane visualization and advances in the field of automation of the fetal heart [7].

\section{Three-Dimensional Color Doppler Rendering}

Color flow Doppler, power Doppler, and bidirectional power Doppler can be combined with the STIC tool to acquire volumes including $2 \mathrm{D}$ and color information throughout the cardiac cycle. The image display can be the tomographic, orthogonal, or the $3 \mathrm{D}$ volume rendering mode. The information displayed in volume rendering can include either the color information alone, the gray-scale information alone, or a combination of both, termed "glass body" mode [7]. A special software using a light source can in addition emphasize the depth effect of the blood flow and the surrounding structures and provides an enhanced spatial impression (Fig. 1).

This glass body display with color or power Doppler is of significant help in the demonstration of the heart cycle-related flow events and in identifying great vessel spatial relationship in complex congenital heart disease $[5,7,11]$. An interesting display includes the 4-chamber view showing normal or abnormal flow events during diastole or systole (Fig. 2), but also a cranial view of the great vessels demonstrating their normal or abnormal spatial relationship (Figs. $3,4)$. An additional approach is from the lateral side view of the heart demonstrating the aortic and ductal arches, or at the level of the upper abdomen showing the abdominal vasculature such as the ductus venosus, the inferior vena cava, and their relationship to the descending aorta.

\section{Matrix Probe Use in Fetal Cardiac Assessment}

An emerging field in 3D/4D ultrasound involves the use of an electronic matrix transducer with the ability to acquire volumes with speed and enhanced resolution. This new technology holds promise in fetal echocardiography.

The usual mechanical 3D transducer consists of one line of crystals used to generate the $2 \mathrm{D}$ image. In such a probe, the $3 \mathrm{D}$ or $4 \mathrm{D}$ image is produced by a mechanical motor that sweeps the ultrasound beam, thus generating multiple $2 \mathrm{D}$ planes stacked together to produce the $3 \mathrm{D}$ volume. Therefore, 3D acquisitions are slow, which is acceptable in general obstetric sonography with minimal motion. In fetal echocardiography, however, the high frequency of the fetal heart beat is a main limiting factor for static $3 \mathrm{D} / 4 \mathrm{D}$ acquisitions with mechanical motors. This 

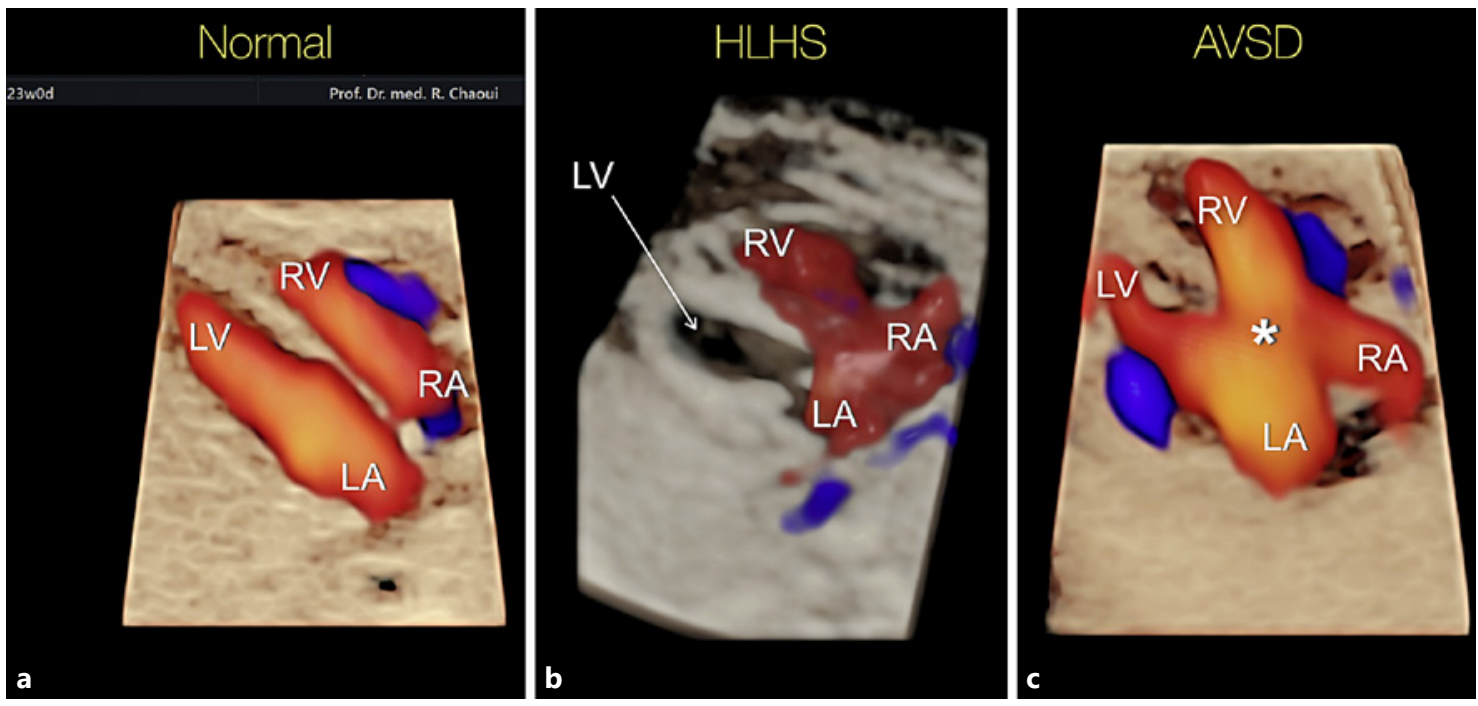

Fig. 2. STIC volume acquisition in color Doppler at the level of the 4-chamber view, displayed in glass-body mode in a normal fetus (a), in a fetus with hypoplastic left heart syndrome (b), and in a fetus with an atrioventricular septal defect (c). b Arrow in shows the hypoplastic left ventricle with absent blood flow across the mi- tral valve. c Asterisk in is placed at the level of the defect in the crux of the heart in atrioventricular septal defect. RA, right atrium; RV, right ventricle; LA, left atrium; HLHS, hypoplastic left heart syndrome; AVSD, atrioventricular septal defect; LV, left ventricle.

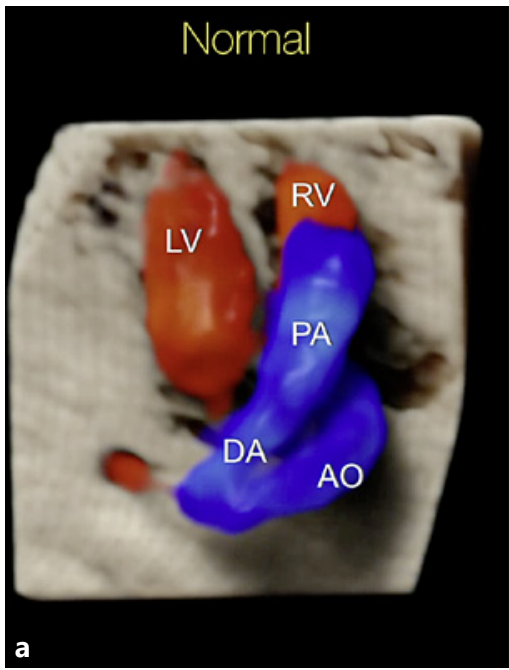

Fig. 3. STIC volume acquisition in color Doppler, displayed in glass-body mode at the level of the 3-vessel-trachea view in a normal fetus (a), in a fetus with hypoplastic left heart syndrome (b), and in a fetus with tetralogy of Fallot (c). Note in (a) the normal course of the pulmonary artery (PA) with ductus arteriosus and the aorta. The dilated PA and DA with antegrade flow (blue) and the diminutive aortic arch with retrograde flow (red color) across
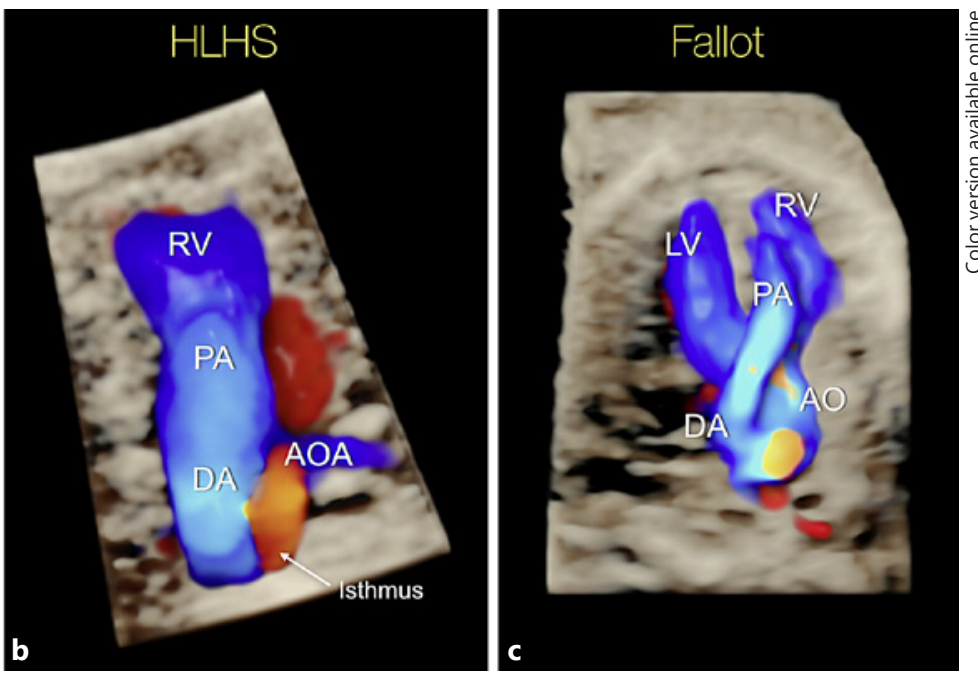

the isthmus in noted in fetus (b). Note the disproportion of the size of the great vessels in fetus (c) with a dilated aorta and a narrow PA, both showing antegrade flow on color Doppler (both blue). Note the size difference in the outflow tracts between fetus (a-c). $\mathrm{LV}$, left ventricle; RV, right ventricle; HLHS, hypoplastic left heart syndrome; PA, pulmonary artery; DA, ductus arteriosus; AOA, aortic arch; AO, aorta. 

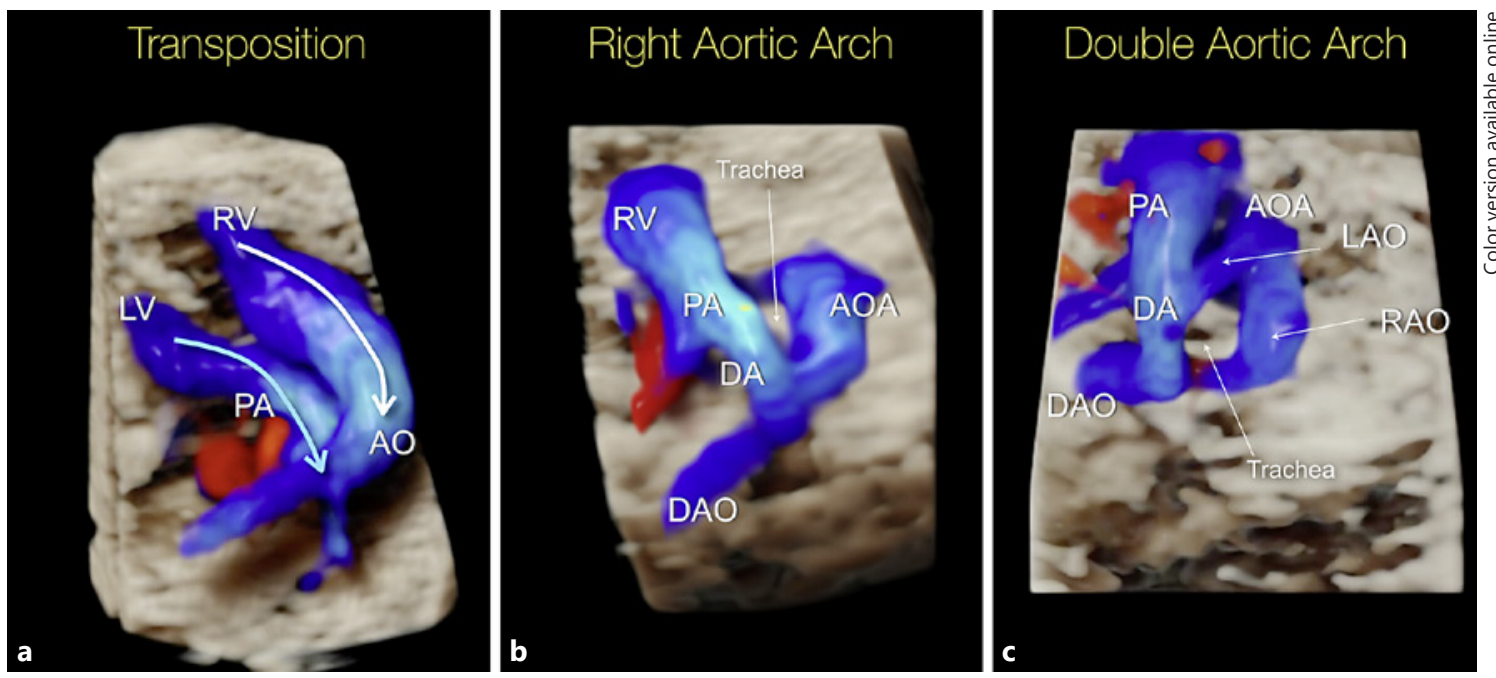

Fig. 4. STIC volume acquisition in color Doppler, displayed in glass-body mode at the level of 3-vessel-trachea view in a fetus with a D-transposition of the great arteries (a), in a fetus with rightsided aortic arch (b) and in a fetus with double aortic arch (c). Note in (a) the parallel course of aorta and pulmonary artery. b Shows

limitation has led to the advent of STIC acquisition as a good compromise between high frame rates of a fetal heart and low acquisition time.

The recent advent of electronic matrix transducers enables a more rapid examination of the heart in $3 \mathrm{D} / 4 \mathrm{D}$. These matrix array transducers are designed with multiple rows of crystals (64 rows in some), thus creating a transducer footprint with $>8,000$ elements. With the high computing speed, matrix transducers are able to electronically steer the ultrasound beam through a region of interest and to acquire $3 \mathrm{D}$ volumes up to 4 times faster than a conventional mechanical 3D transducer. The improved resolution of the $3 \mathrm{D}$ volumes acquired by the matrix transducers is reflected in the good quality of the acquisition but also of the reconstructed images within the volume. Two main applications of matrix transducers are the ability to get 2 instantaneous orthogonal planes (called biplane or X-plane) and the real-time $4 \mathrm{D}$ visualization of the fetal heart including the rapid acquisition of STIC volumes. Some of these aspects are discussed here.

\section{Real-Time 4D and Rapid STIC}

An exciting aspect of the matrix probe is its ability to scan the heart in real-time $4 \mathrm{D}$ mode. Choosing an appropriate preset with a narrow acquisition volume box enables a live $4 \mathrm{D}$ fetal cardiac examination with frame rates be- the trachea between the aortic arch (AOA) and pulmonary artery. c Shows the AOA bifurcating into a left and a right AOA surrounding the trachea and creating a vascular ring. DAO, descending aorta; $L V$, left ventricle; RV, right ventricle; $P A$, pulmonary artery; $\mathrm{AOA}$, aortic arch; LAO, left aortic; RAO, right aortic; $\mathrm{AO}$, aorta.

tween 20 and 40 volumes per second (Fig. 5), especially in the second and third trimester. The image display can be in the orthogonal or tomographic mode, and also in 3D rendering with the surface mode (Fig. 5), inversion mode, or others [11]. Color Doppler can also be used in combination with real-time $4 \mathrm{D}$ mode, but this acquisition time is significantly slow and has a reduced contribution to diagnostic use. Another application is the ability to rapidly acquire a STIC volume, typically within $2-3$ s called electronic STIC (eSTIC) or rapid STIC. This approach is ideal for color Doppler volumes and for artifacts reduced volumes.

The eSTIC images consist of subvolumes that are stitched together. This increases the speed of acquisition. In addition, unlike the mechanical probe in which increasing the distance traveled decreases the image quality, with the eSTIC, the distance traveled is no longer relevant. The size of the matrix footprint of each of the subvolumes provides almost "artifact-free images" within each subvolume.

During the 2nd trimester acquisition, the user may have 1-3 subvolumes. The benefit is each subvolume contains artifact-free images in the $\mathrm{B}$ and $\mathrm{C}$ planes.

\section{Biplane $^{\circledR}$ or Live-X-Plane ${ }^{\circledR}$}

The simultaneous acquisition of many images using a matrix probe allows for the real-time display of 2 orthogonal 2D planes with high frame rate and good resolution 

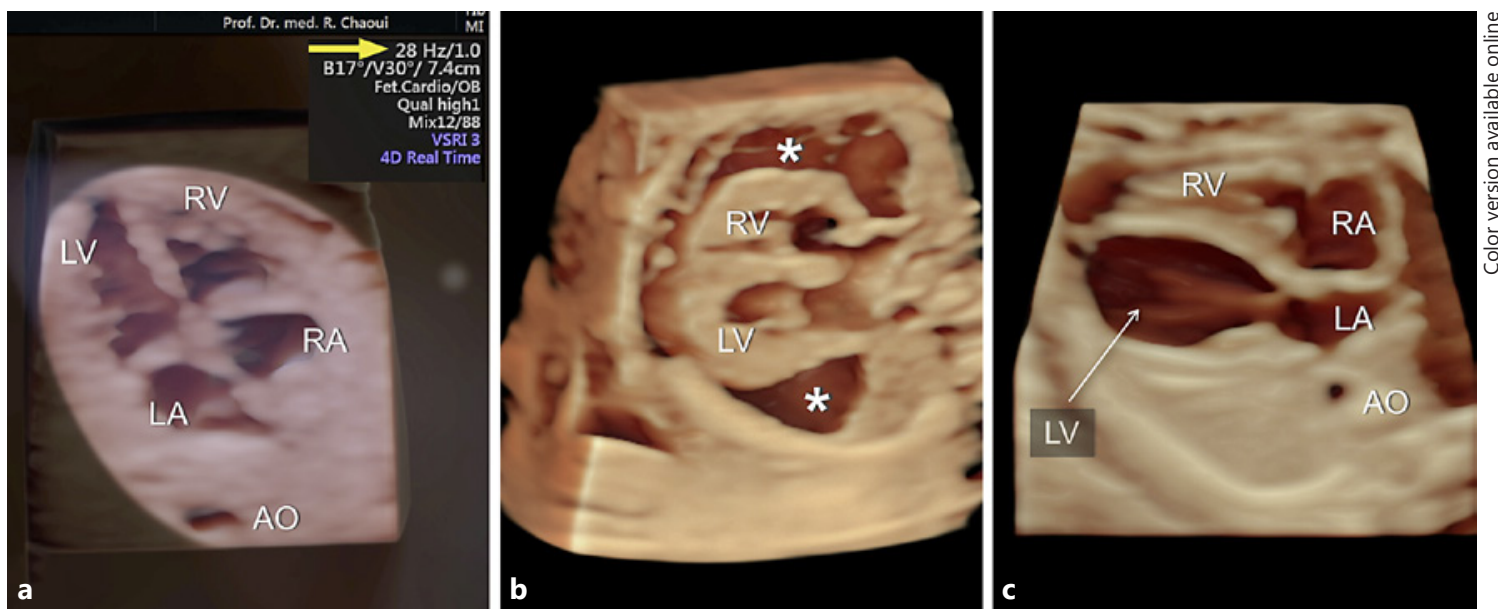

Fig. 5. Surface mode rendering display of a matrix array transducer in real-time 4D mode at the 4-chamber view of the fetal heart in 3 fetuses (panels a-c). a Shows a normal heart. Note in (a) the frame rate of 28 volumes per second (arrow). b Shows a fetus with cardiomyopathy and large pericardial effusion (asterisks). c Shows a fetus with a dilated left ventricle, a sign of left ventricular dysfunction in critical aortic stenosis. AO, aorta, LA, left atrium, RA, right atrium; RV, right ventricle; $L V$, left ventricle.

Fig. 6. Biplane mode display of an electronic matrix array transducer. The scanning plane, seen in (a), shows an axial view of the upper mediastinum at the level of the 3 -vessel-trachea view demonstrated the pulmonary artery and aortic isthmus (Ao). Activating the biplane mode allows the examiner to place a line along the image (dotted line in a), which generates an orthogonal plane (b) to the scanning plane (a) in real-time imaging. Note the longitudinal view of the aortic arch in (b), which represents the orthogonal plane to the aortic isthmus shown in (a) along the dotted line (arrow heads). SVC, superior vena cava; $\mathrm{PA}$, pulmonary artery; AO, aorta.

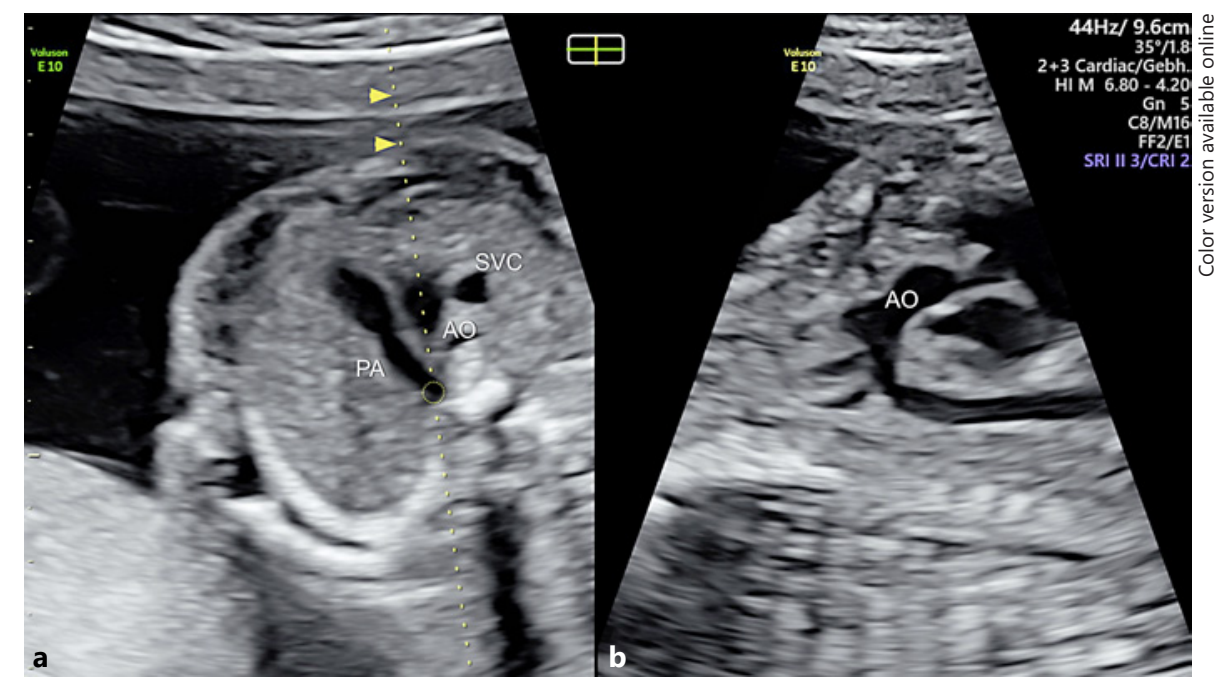

(Figs. 6, 7), without the need to acquire a 4D volume. The live-X-plane ${ }^{\circledR}[12]$ or biplane ${ }^{\circledR}$ provides instantaneous display of 2 perpendicular planes, where one is the actual scanning plane and the other is the orthogonal plane to it. This new tool can be used in 2D (Figs. 6, 7) or in combination with color Doppler (Fig. 8). Main applications of the live-x-plane ${ }^{\circledR}$ or biplane ${ }^{\circledR}$ mode in the examination of the fetal heart include the visualization of the interventricular septum [13] in order to demonstrate or rule out septal defects in 2 orthogonal planes (Fig. 8) as well as the ability to image the great vessels in 2 simultaneous orthogonal planes, which allows for the depiction of normal cardiac anatomy and for the demonstration of abnormal great vessel relationship in conotruncal anomalies (Fig. 7) [14].

\section{Volume Sonography and the Automation of the Fetal Heart Examination}

Despite advancement in ultrasound imaging, prenatal detection of congenital heart disease is still low based upon population studies. The complex anatomy of the fetal heart along with its small size and the diverse nature 
Fig. 7. Biplane mode display in a fetus with a transposition of the great arteries. The scanning plane, seen in (a), shows an axial view of the upper mediastinum at the level of the 3-vessel-trachea view demonstrated the aorta as the single great vessel anterior to the pulmonary artery. The orthogonal plane displayed in (b) shows the aorta and pulmonary artery in longitudinal view in parallel course to each other's. SVC, superior vena cava; $\mathrm{PA}$, pulmonary artery; $\mathrm{AO}$, aorta.

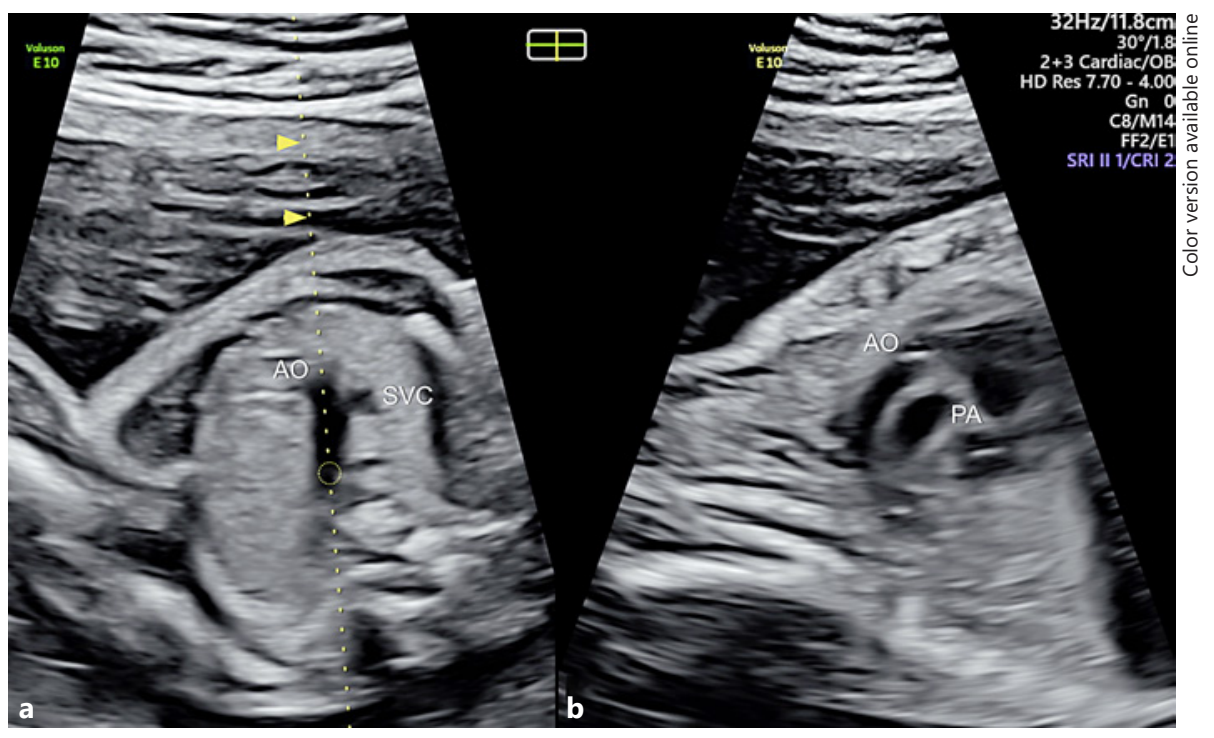

Fig. 8. Biplane mode display in color Doppler of a fetus with a muscular ventricular septal defect with blood flow across the ventricular septal defect is seen in both planes. The dotted line is placed along the interventricular septum in a 4-chamber view (a). An "en-face" view of the area of the interventricular septum is displayed in the (b). LA, left atrium, LV, left ventricle, $\mathrm{RA}$, right atrium, $\mathrm{RV}$, right ventricle; $\mathrm{VSD}$, ventricular septal defect.

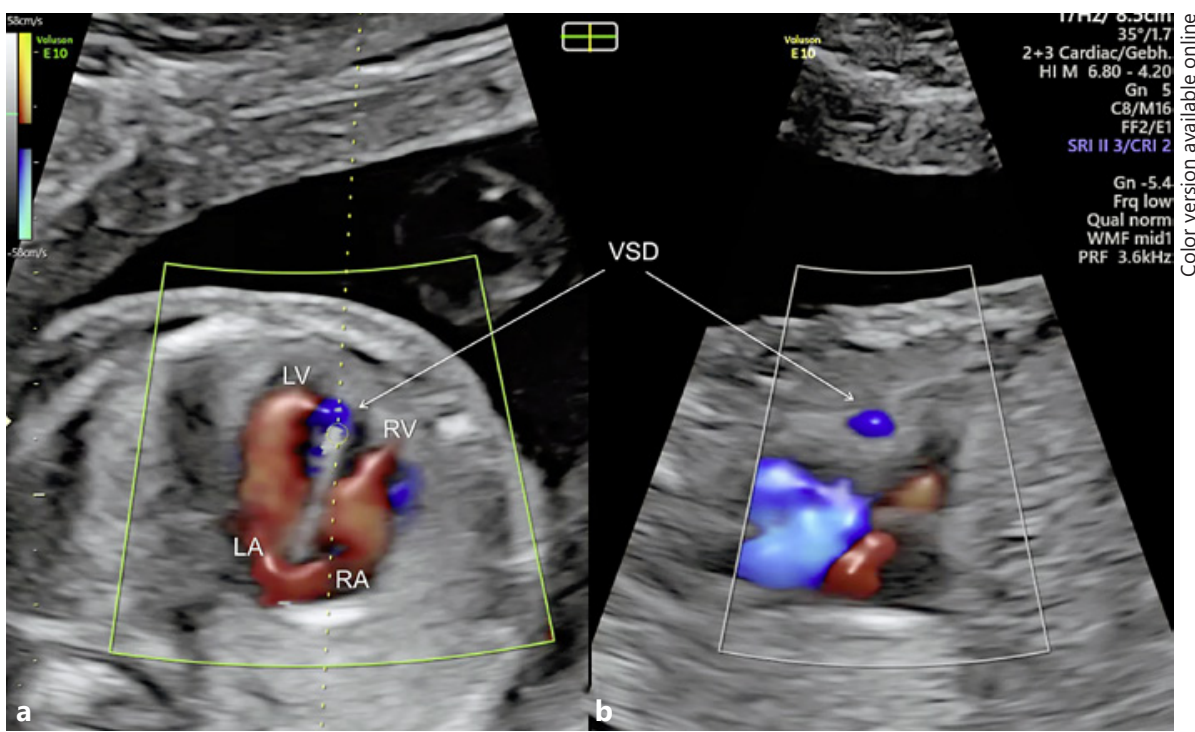

of CHD adds to the complexity of the fetal heart examination. In addition, the operator dependency of ultrasound, along with the variable position of the fetus within the abdomen, results in lack in standardization, consistency, and reproducibility. 3D/4D ultrasound along with STIC technology has become prevalent in the last decade, and this technology has been used to improve the screening examination of the fetal heart. It should be borne in mind that a $3 \mathrm{D}$ volume data set contains all the $2 \mathrm{D}$ planes needed for a complete anatomic evaluation, and the main advantage of volume ultrasound is the ability to extract and display diagnostic $2 \mathrm{D}$ planes from a 3D volume. This led our group $>10$ years ago to work on the automated ex- amination of the fetal heart using $3 \mathrm{D}$ volumes $[15,16]$. An important aspect to emphasize is that for any organ, such as the fetal heart, the anatomic planes of interest are organized in a constant anatomic spatial relationship to each other. Data on the topographic orientation of these planes can be used to develop algorithms, which allows for the automated examination of the heart using 3D, STIC, eSTIC, or 4D volumes [11].

Our group was the first to introduce the automation software into fetal volume ultrasound and initially termed this approach as automated multiplanar imaging $[15,16]$. This later evolved into Sono-VCAD for Volume Computer-Aided Diagnosis with a tomographic display of the 

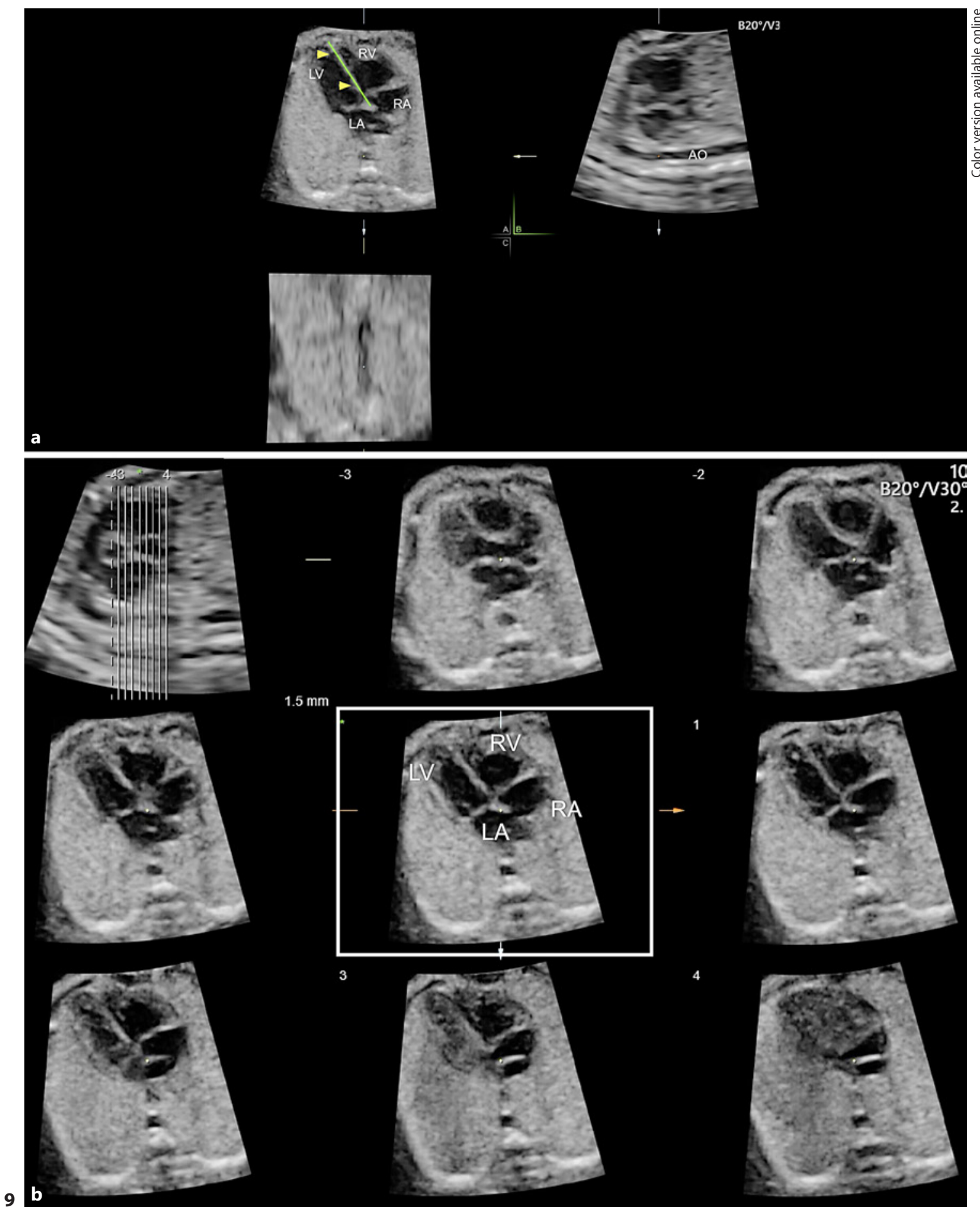

(For figure $9 \mathrm{c}$ and legend see next page.)

retrieved planes [16] and was then implemented into commercially available ultrasound systems (Voluson Equipment, General Electric Healthcare, Zipf-Austria). Later another group introduced a similar approach called for Fetal Intelligent Navigation Echocardiography, which is used on another commercially available ultrasound systems (WS80, Samsung Healthcare, Seoul, Korea) [17, 18]. The principle in both software is similar: The volume of the heart has to be acquired as a 4-chamber view ideally from an apical insonation with the fetus lying in a dorso- 


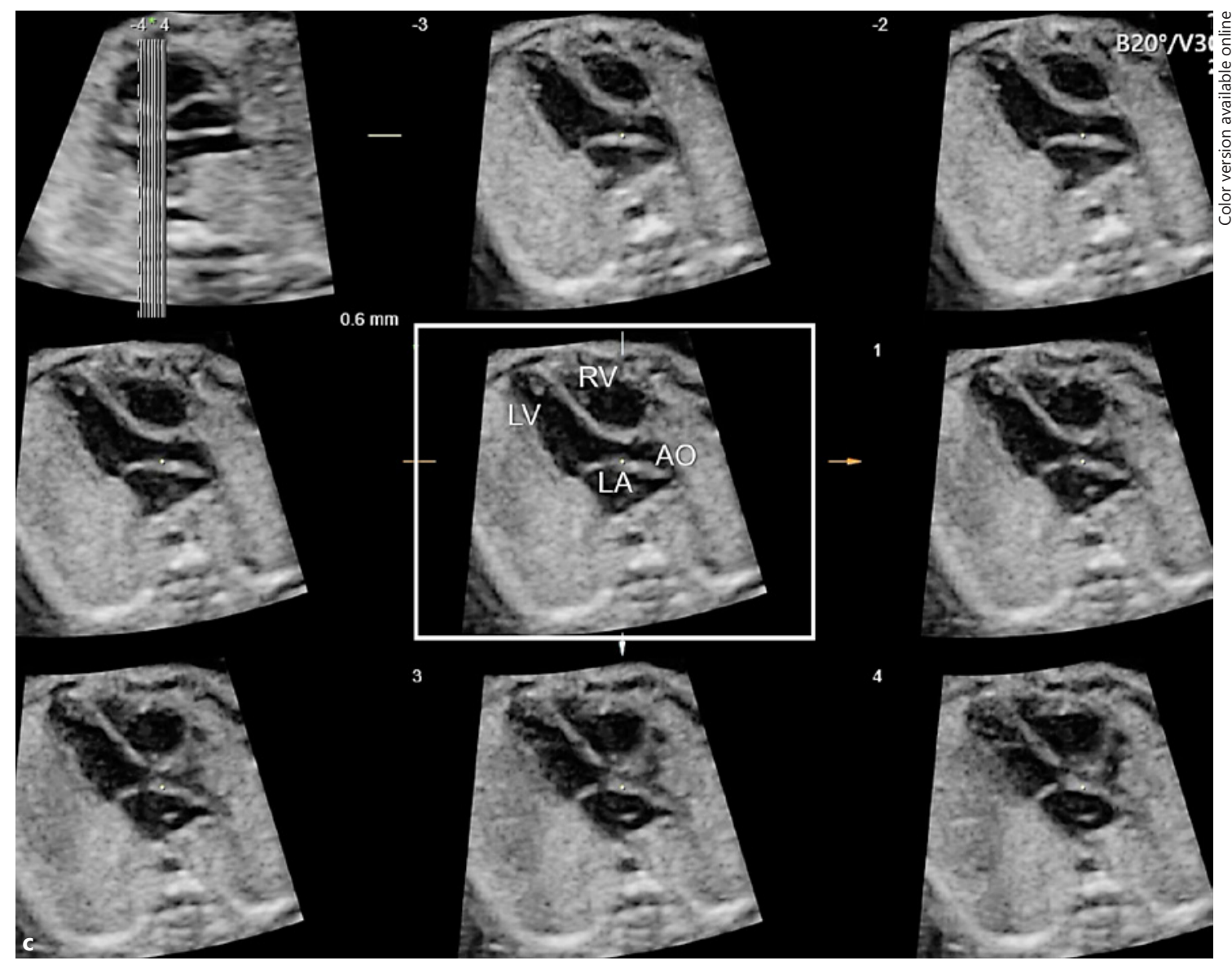

Fig. 9. a Standardization of a 3D volume of the fetal chest obtained at the level of the 4-chamber view for the SonoVCAD software application. Note that standardization is performed by tracing a line along the interventricular septum (arrowheads and green line) and localizing the DAO (white reference dot). Standardization of a 3D volume is the first step for SonoVCAD automation. See next steps in $\mathbf{b}$ and $\mathbf{c}$. $\mathbf{b}$ SonoVCAD applied to the acquired 3D volume of the fetal chest, shown in a. After localizing the septum and the DAO, the automation software displays the 4-chamber plane as the standard plane (white square). The display is presented in tomographic view with multiple planes in order to account for subtle variabil- ity between fetuses. c SonoVCAD applied to the acquired 3D volume of the fetal chest, shown in $\mathbf{a}$ and $\mathbf{b}$. After the 4-chamber view is recognized as the standard plane (b), the examiner can choose to automatically display one or more diagnostic planes of the fetal heart, the left ventricular outflow tract (white square) in the demonstrated display. Similar to the 4-chamber view, the display of the left ventricular outflow tract is presented in tomographic view with multiple planes in order to account for subtle variability between fetuses. AO, aorta; LA, left atrium; LV, left ventricle; RA, right atrium; RV, right ventricle. posterior position [19]. The examiner defines some specific structures on the volume data set (septum, descending aorta, and others) to confirm the volume orientation (Fig. 9a). The automation software then extracts the conventional diagnostic planes out of the volume (Fig. 9b, c) [19]. It has been shown that these techniques can simplify the examination of the fetal heart and reduce operator dependency [19]. We have to keep in mind that the software is mainly able to display the diagnostic ultrasound planes and the operator should decide whether these are normal or abnormal. In a recent opinion on this subject, 4 limitations of the technique were emphasized [19]: (1) the result depends mainly on the quality of the acquired volume data set, which may underlie artifacts, (2) the software is optimized for the core examination interval of mid-pregnancy, around 20 weeks, and first and third trimester may not lead to satisfactory results, (3) the software is based upon the hypothesis that there is normal cardiac anatomy and that structures of interest are present in their usual anatomic location, and (4) the operator must be able to mark the anatomical structures properly as a prerequisite for automation software to extract the 
planes [19]. We believe that the $3 \mathrm{D} / 4 \mathrm{D}$ technology will allow us to move closer to near-full automation of the cardiac examination with minimal operator dependency. This should prove to be a giant step forward toward the simplification of the cardiac screening examination and a very important step toward optimal screening of the fetal heart. It is also expected that in the future, image recognition software will enhance the ability to recognize normal and abnormal fetal cardiac structures and help triage atrisk pregnancies to expert fetal echocardiography.

\section{Conclusions}

3D/4D ultrasound provided important advancement in fetal echocardiography, which allowed for enhanced image acquisition and display and for the application of post-processing tools to help with spatial display of cardiac structures. The advent of the matrix probe promises an improvement in imaging as it enables an almost live
$4 \mathrm{D}$ examination of the fetal heart, a rapid STIC acquisition (eSTIC) with reduced artifacts [20], as well as new tools as biplane or $\mathrm{x}$-plane with a new perspective of the cardiac examination. The ability to semi-automate the fetal heart examination will ultimately result in simplification of the fetal echocardiography examination, with the ultimate goal of improving detection of congenital heart disease. As the ultrasound technology evolves, novel applications will undoubtedly be incorporated in the fetal heart examination and hopefully result in standardization and optimization of fetal echocardiography.

\section{Disclosure Statement}

R.C. and K.S.H. collaborate in testing new software for the Voluson series with GE-Healthcare. A.A. collaborates in testing new software for the Voluson series with GE-Healthcare and developed the basics of the Sono-VCAD software. Tools presented in this article are not exclusively related to one product but to products commercially available from different companies.

\section{References}

1 Lee W, Allan L, Carvalho JS, Chaoui R, Copel J, Devore G, et al.; ISUOG Fetal Echocardiography Task Force. ISUOG consensus statement: what constitutes a fetal echocardiogram? Ultrasound Obstet Gynecol. 2008 Aug; 32(2):239-42.

2 Carvalho JS, Allan LD, Chaoui R, Copel JA, DeVore GR, Hecher K, et al.; International Society of Ultrasound in Obstetrics and Gynecology. ISUOG Practice Guidelines (updated): sonographic screening examination of the fetal heart. Ultrasound Obstet Gynecol. 2013 Mar;41(3):348-59.

3 DeVore GR, Falkensammer P, Sklansky MS, Platt LD. Spatio-temporal image correlation (STIC): new technology for evaluation of the fetal heart. Ultrasound Obstet Gynecol. 2003 Oct;22(4):380-7.

4 DeVore GR, Polanco B, Sklansky MS, Platt LD. The 'spin' technique: a new method for examination of the fetal outflow tracts using three-dimensional ultrasound. Ultrasound Obstet Gynecol. 2004 Jul;24(1):72-82.

5 Chaoui R, Hoffmann J, Heling KS. Three-dimensional (3D) and 4D color Doppler fetal echocardiography using spatio-temporal image correlation (STIC). Ultrasound Obstet Gynecol. 2004 Jun;23(6):535-45.

6 Paladini D, Vassallo M, Sglavo G, Lapadula C, Martinelli P. The role of spatio-temporal image correlation (STIC) with tomographic ultrasound imaging (TUI) in the sequential analysis of fetal congenital heart disease. Ultrasound Obstet Gynecol. 2006 May;27(5): $555-61$.
7 Chaoui R, Heling KS. 3D-ultrasound in prenatal diagnosis: a practical approach. 1st edition. Berlin, New York: DeGruyter; 2016.

8 Viñals F. Current experience and prospect of internet consultation in fetal cardiac ultrasound. Fetal Diagn Ther. 2011;30(2):83-7.

9 Espinoza J, Lee W, Comstock C, Romero R, Yeo L, Rizzo G, et al. Collaborative study on 4-dimensional echocardiography for the diagnosis of fetal heart defects: the COFEHD study. J Ultrasound Med. 2010 Nov;29(11):1573-80.

10 Hamill N, Yeo L, Romero R, Hassan SS, Myers SA, Mittal P, et al. Fetal cardiac ventricular volume, cardiac output, and ejection fraction determined with 4-dimensional ultrasound using spatiotemporal image correlation and virtual organ computer-aided analysis. Am J Obstet Gynecol. 2011 Jul;205(1):76.e1-10.

11 Abuhamad A, Chaoui R. A practical guide to fetal echocardiography: normal and abnormal hearts. 3rd ed. Philadelphia (PA): Wolters Kluwer Health/Lippincott Williams \& Wilkins; 2015.

12 Xiong Y, Chen M, Chan LW, Ting YH, Fung TY, Leung TY, et al. A novel way of visualizing the ductal and aortic arches by real-time three-dimensional ultrasound with live xPlane imaging. Ultrasound Obstet Gynecol. $2012 \operatorname{Mar} ; 39(3): 316-21$

13 Xiong Y, Liu T, Wu Y, Xu JF, Ting YH, Yeung Leung $\mathrm{T}$, et al. Comparison of real-time threedimensional echocardiography and spatiotemporal image correlation in assessment of fetal interventricular septum. J Matern Fetal Neonatal Med. 2012 Nov;25(11):2333-8.
14 Xiong Y, Liu T, Gan HJ, Wu Y, Xu JF, Ting $\mathrm{YH}$, et al. Detection of the fetal conotruncal anomalies using real-time three-dimensional echocardiography with live xPlane imaging of the fetal ductal arch view. Prenat Diagn. 2013 May;33(5):462-6.

15 Abuhamad A, Falkensammer P, Zhao Y. Automated sonography: defining the spatial relationship of standard diagnostic fetal cardiac planes in the second trimester of pregnancy. J Ultrasound Med. 2007 Apr;26(4):501-7.

16 Abuhamad A, Falkensammer P, Reichartseder F, Zhao Y. Automated retrieval of standard diagnostic fetal cardiac ultrasound planes in the second trimester of pregnancy: a prospective evaluation of software. Ultrasound Obstet Gynecol. 2008 Jan;31(1):30-6.

17 Yeo L, Luewan S, Markush D, Gill N, Romero R. Prenatal Diagnosis of Dextrocardia with Complex Congenital Heart Disease Using Fetal Intelligent Navigation Echocardiography (FINE) and a Literature Review. Fetal Diagn Ther. 2018;43(4):304-16.

18 Veronese P, Bogana G, Cerutti A, Yeo L, Romero R, Gervasi MT. A Prospective Study of the Use of Fetal Intelligent Navigation Echocardiography (FINE) to Obtain Standard Fetal Echocardiography Views. Fetal Diagn Ther. 2017;41(2):89-99.

19 Yeo L, Romero R. Intelligent navigation to improve obstetrical sonography. Ultrasound Obstet Gynecol. 2016 Apr;47(4):403-9.

20 DeVore GR, Satou G, Sklansky M. 4D fetal echocardiography-An update. Echocardiography. 2017 Dec;34(12):1788-98. 